\title{
Magnetic collimation of fast electrons produced by ultraintense laser irradiation by structuring the target composition
}

\author{
A. P. L. Robinson \\ Central Laser Facility, STFC Rutherford-Appleton Laboratory, Chilton, Didcot, Oxfordshire, OX11 OQX, \\ United Kingdom \\ M. Sherlock \\ Central Laser Facility, STFC Rutherford-Appleton Laboratory, Chilton, Didcot, Oxfordshire, OX11 OQX, \\ United Kingdom and SUPA, Department of Physics, University of Strathclyde, Glasgow, G4 ONG, \\ United Kingdom
}

(Received 5 June 2007; accepted 11 July 2007; published online 29 August 2007)

\begin{abstract}
A scheme for collimating fast electrons in a specially engineered solid target is proposed. Unlike previous approaches, the collimation is achieved by generating an azimuthal magnetic field as opposed to a radial electric field. The target is engineered such that it consists of a fiber surrounded by material of a lower resistivity than that of the fiber. The fast electrons are collimated along the fiber. Hybrid Vlasov-Fokker-Planck simulations supported by analytic calculations show that this concept is viable. (C) 2007 American Institute of Physics. [DOI: 10.1063/1.2768317]
\end{abstract}

\section{INTRODUCTION}

When relativistically intense $\left(>10^{18} \mathrm{~W} \mathrm{~cm}^{-2} \mu \mathrm{m}^{2}\right)$ lasers interact with solid targets, beams of relativistic electrons are produced. It has been proposed that such a beam could be used as an ignitor beam in a fast ignition (FI) inertial confinement fusion scheme. ${ }^{1,2}$ The generation of $\mathrm{x}$ rays as the fast electron beam propagates through solid material means that laser-foil interactions are, potentially, an excellent source for radiographic applications. ${ }^{3}$ The importance of fast electron transport to proton/ion acceleration is also well recognized. ${ }^{4-6}$ This has led to a large number of experimental ${ }^{7-15}$ and theoretical ${ }^{16-23}$ studies of fast electron transport.

All of these uses of fast electrons ideally demand a well collimated beam of fast electrons. For fast ignition this is crucial to the energetic viability of the scheme. For radiographic applications, this determines the source size. However, a number of experimental results suggest that, at intensities above $10^{19} \mathrm{~W} \mathrm{~cm}^{-2}$, the fast electron beam radius grows to several times the laser focal spot radius (see Refs. 24 and 25) rather than collimating to a small radius. Although the fast electron beam might "naturally" be expected to resistively pinch in the solid density plasma, the work of Bell and Kingham ${ }^{26}$ detailed a number of factors that can prevent this. Most importantly, natural collimation becomes increasingly less likely the larger the initial divergence angle of the fast electron beam. Natural collimation is also less favoured by higher laser powers and fast electron temperatures.

This motivated a number of studies into the collimation of fast electron beams by "artificial" means. By this we mean engineering the laser pulse or target to induce collimation under conditions in which natural, resistive collimation would not otherwise occur. Thus far, such research efforts have concentrated on using radial electric fields to collimate the fast electrons, and this naturally leads to employing radial vacuum gaps, as in the work of Campbell et al., ${ }^{27}$ or to simply limiting the target to being a wire, as in the work of Kodama $e t \mathrm{al}^{28}$ In this paper we propose engineering the target to artificially induce magnetic collimation of the fast electron beam. This is achieved by structuring the target so that the target consists of a thin fiber embedded in material that is less resistive than the fiber. An azimuthal magnetic field is generated around the fiber, and the fast electrons are collimated along the fiber. It is shown that the fast electrons are strongly collimated down to beam radii comparable to the laser focal spot radius.

The paper is structured as follows: In Sec. II we explain the theory of this concept. In Sec. III we describe the numerical code used, and in Secs. IV and V we present the simulation results.

\section{THEORY}

The generation of magnetic fields during the propagation of fast electrons through a solid target can be described by combining a simple Ohm's law $\left(\mathbf{E}=-\eta \mathbf{j}_{\text {fast }}\right)$ with Faraday's law to yield

$$
\frac{\partial \mathbf{B}}{\partial t}=\eta \nabla \times \mathbf{j}_{f}+\nabla(\eta) \times \mathbf{j}_{f},
$$

where $\eta$ is the resistivity, and $\mathbf{j}_{f}$ is the fast electron current density. Davies ${ }^{29}$ noted that there is a simple qualitative interpretation of Eq. (1). The first term on the right-hand side generates a magnetic field that pushes fast electrons towards regions of higher fast electron current density, while the second term pushes fast electrons towards regions of higher resistivity. It is this second term that the proposed concept exploits, although it will be seen that the other term is also important. This qualitative interpretation immediately implies that, if a target consists of a fiber surrounded by material that is less resistive than the fiber, then a magnetic field will be generated that will collimate the fast electrons along the fiber. A quantitative estimate of the degree of target structuring that is required to actually achieve this can be ob- 
tained by employing Davies' "rigid beam" model to estimate the magnetic field generation. In this one-dimensional model, an axial fast electron current density is imposed, i.e.,

$$
j_{f}=j_{0} \exp \left(-r^{2} / 2 R_{f}^{2}\right),
$$

where $R_{f}$ is the fast electron beam radius. The fast electron beam is assumed to remain static or "rigid." The azimuthal magnetic field generation is then obtained from Eq. (1). For this calculation we assumed a Spitzer resistivity and ideal gas specific heat capacity, i.e.,

$$
\eta=10^{-4} Z \ln \Lambda / T_{c}^{3 / 2},
$$

where $T_{c}$ is the temperature of the cold electrons (in eV), and

$$
C=\frac{3}{2} n_{c},
$$

where $n_{c}$ is the density of the cold electrons. The model is completed by an equation for the Ohmic heating of the background plasma:

$$
\frac{\partial T_{c}}{\partial t}=\frac{\eta j_{f}^{2}}{C} .
$$

The results of a numerical integration are presented in Fig. 1. In this calculation, the initial cold electron temperature was set at $200 \mathrm{eV}$, and the cold electron density was set to $6 \times 10^{28} \mathrm{Z} \mathrm{m}^{-3}$. For the fast electron beam, we set $j_{0}=5$ $\times 10^{15} \mathrm{~A} \mathrm{~m}^{-2}$, and $R_{f}=10 \mu \mathrm{m}$. To impose some target structure, the background $Z$ was varied by $Z=3$ $+7 \exp \left(-r^{2} / 2 R_{Z}^{2}\right)$, where we set $R_{Z}=5 \mu \mathrm{m}$. The Coulomb logarithm was taken to be 2 . This calculation shows that magnetic flux densities of over $100 \mathrm{~T}$, over a radial range of approximately $10 \mu \mathrm{m}$, can be achieved by structuring the $\operatorname{target} Z$ this way. By considering the orbit of an electron in a uniform magnetic field of flux density $B_{0}$ with extent $L$, one finds that the electron is reflected back towards the axis if

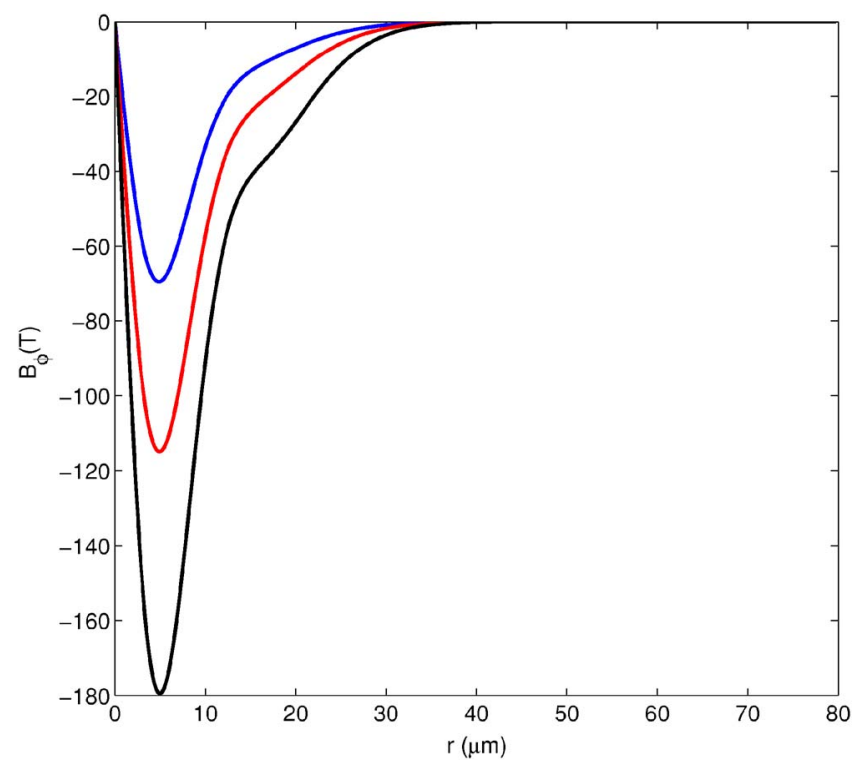

FIG. 1. (Color online) Magnetic field in "rigid beam" model calculation at $125 \mathrm{fs}$ (top curve), $250 \mathrm{fs}$ (middle curve), and $500 \mathrm{fs}$ (bottom curve).

$$
B_{0} L>\frac{\gamma_{f} v_{f} m_{e}}{e}(1-\cos \theta),
$$

where $v_{f}$ is the speed of the fast electron, $\gamma_{f}$ is its Lorentz factor, and $\theta$ is the angle the fast electron makes to the target axis on entering the $B$-field. From Eq. (6), one finds that the fields generated in the rigid beam calculation are sufficient to reflect $1.8 \mathrm{MeV}$ fast electrons that enter at an angle of $\theta=30^{\circ}$.

The conclusion that should be drawn from this is that it is possible to generate a significant collimating magnetic field by structuring the background resistivity. However, this alone will not be sufficient to fully collimate the fast electron beam in the case of multi-MeV fast electrons and large divergence angles (i.e., $>30^{\circ}$ ). On the other hand, this field may initiate collimation, which will then be greatly enhanced by further magnetic field generation due to the $\eta \boldsymbol{\nabla} \times \mathbf{j}_{f}$ term. Therefore, on the basis of this analytic model alone, it is not absolutely certain that artificial collimation will occur. Detailed numerical simulation is required to clarify matters.

\section{NUMERICAL CODE}

The numerical code used is the LEDA code, which is a two-dimensional hybrid Vlasov-Fokker-Planck (VFP) code. By this we mean a code that uses a VFP (specifically a KALOS-like ${ }^{26}$ ) algorithm to describe the fast electrons, while the background electrons are given a hybrid description as in the hybrid code of Davies. ${ }^{30}$ The distribution function of the fast electrons is expressed as a (truncated) spherical harmonic expansion with terms of the form $f_{l}^{m}(x, y, p) P_{l}^{m}(\cos \theta) \exp (i m \phi)$. Substituting this expansion into the VFP equation yields a set of equations for the $f_{l}^{m}(x, y, p)$ coefficients. The solution of these equations through the KALOS algorithm is explained to a certain extent in Refs. 26 and 31. LEDA utilizes a robust characteristic based method for the $x$-advection that was previously employed in the code in Ref. 32. For the $p$-advection and $y$-advection, Runge-Kutta methods are employed. The electron motion due to the magnetic field is done implicitly, as is the angular scattering of fast electrons off background ions. All of the spatial boundaries are reflective.

The hybrid background is described by an expression for the resistivity and the specific heat capacity. The electric field is then determined by the simple Ohm's law, i.e., $\mathbf{E}=-\eta \mathbf{j}_{\text {fast }}$, and the magnetic field by Eq. (1). The background temperature evolves according to an energy equation [i.e., Eq. (5)]. Thermal conduction is not included. The code also has the option to include the electron pressure term, i.e., $-\nabla p_{c} / n_{c}$, in Ohm's law, and this was used in run I only (see Sec. IV). In order to structure the target, the composition was varied between two materials according to $n_{i}=n_{i, 1} \psi+n_{i, 2}(1-\psi)$ and $Z=Z_{1} \psi+Z_{2}(1-\psi)$. Two forms were used for $\psi$, the first being

$$
\psi=\left\{\begin{aligned}
\exp \left[-\left(y-y_{m}\right) / 2 \sigma_{y}^{2}\right]: & x>x_{0}, \\
1: & x \leqslant x_{0},
\end{aligned}\right.
$$

where $y_{m}=y_{\max } / 2$ (i.e., line parallel to the $x$ axis through the center of the grid), and 


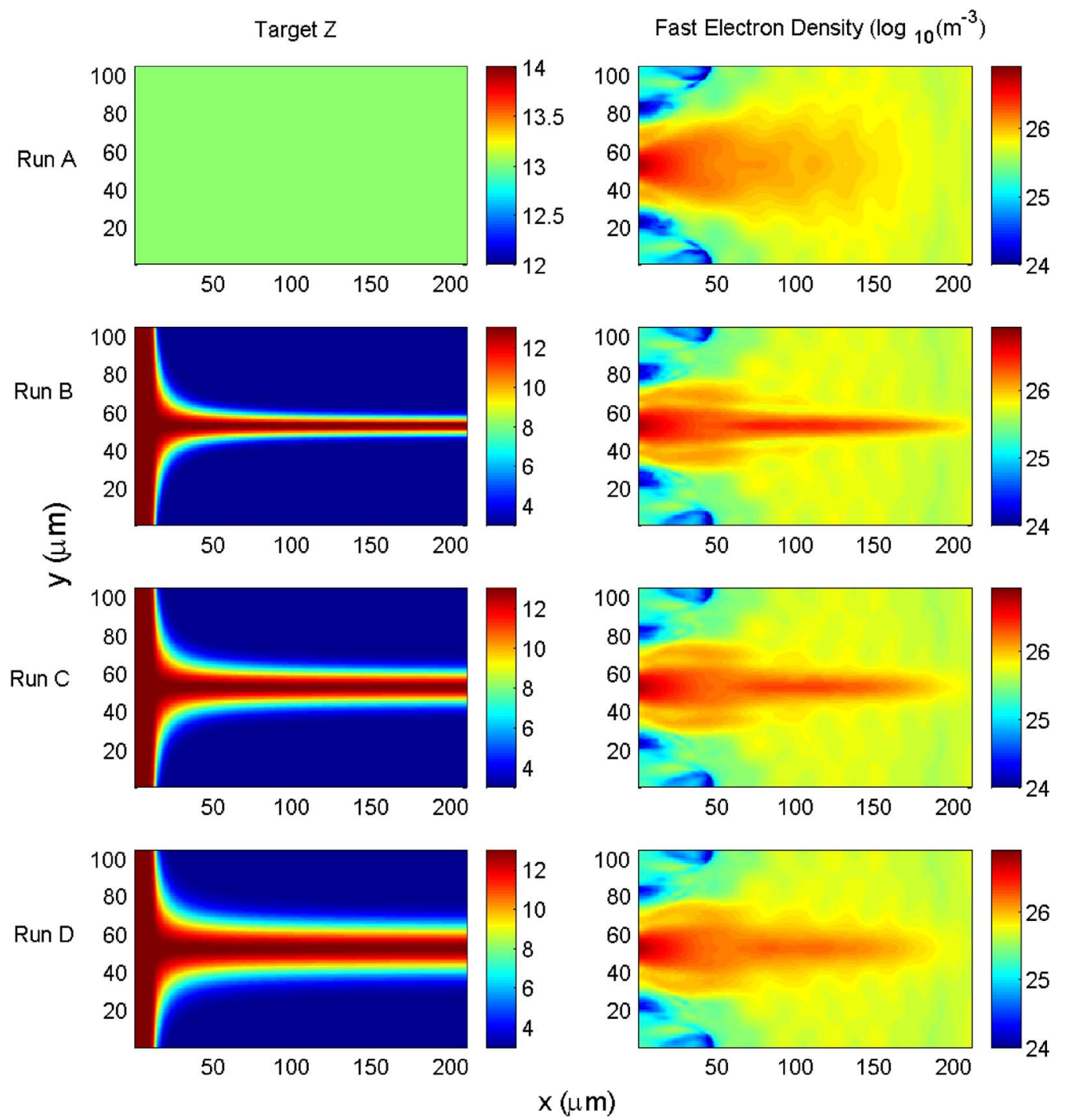

FIG. 2. (Color online) Target $Z$ (left column) and fast electron density at 750 fs (right column) in runs A-D (top-bottom). Run A is the control run, and runs B-D have the background composition determined by Eq. (7). In runs B-D, the fiber width is increasing.

$$
\sigma_{y}=\frac{A}{x-x_{0}}+B .
$$

The second form is

$$
\psi=\left\{\begin{array}{rr}
\frac{1}{2}\left\{1-\tanh \left[\left(y-y_{m}-\sigma_{y}\right) / W\right]\right\}: & x>x_{0}, y>y_{m}, \\
\frac{1}{2}\left\{1+\tanh \left[\left(y-y_{m}+\sigma_{y}\right) / W\right]\right\}: & x>x_{0}, \quad y<y_{m}, \\
1: & x \leqslant x_{0},
\end{array}\right.
$$

where $\sigma_{y}$ is given by Eq. (8). The reader may find the plots of the target $Z$ (which have been included in Sec. IV in Figs. 2 and 5) helpful.

Two resistivity models were used in this investigation. The first is a Spitzer resistivity model, where $\eta$ $=10^{-4} Z \ln \Lambda / T_{c}^{3 / 2}$. The specific heat capacity is that of an ideal gas. This is a good approximation in the case where the initial temperature is greater than $100 \mathrm{eV}$, and materials are only specified through $Z \ln \Lambda$. The second resistivity model concerns $\mathrm{Al}$ and $\mathrm{Li}$ (the two materials modelled in the simu- lations) in both the Spitzer regime and at low temperatures. For Al, the fit to the Milchberg results that Davies uses ${ }^{30}$ is employed:

$$
\eta=\frac{T}{5 \times 10^{6}+170 T^{5 / 2}+3 \times 10^{5} T} .
$$

TABLE I. Geometric parameters employed in runs B-H.

\begin{tabular}{lcccc}
\hline \hline Run & $A\left(\mu \mathrm{m}^{2}\right)$ & $B(\mu \mathrm{m})$ & $x_{0}(\mu \mathrm{m})$ & $W(\mu \mathrm{m})$ \\
\hline B & 100 & 2.5 & 10 & $\mathrm{n} / \mathrm{a}$ \\
C & 100 & 5 & 10 & $\mathrm{n} / \mathrm{a}$ \\
D & 100 & 7.5 & 10 & $\mathrm{n} / \mathrm{a}$ \\
E & 100 & 10 & 10 & $\mathrm{n} / \mathrm{a}$ \\
F & 100 & 5 & 10 & 2 \\
G & 100 & 10 & 10 & 2 \\
$\mathrm{H}$ & 100 & 20 & 10 & 2 \\
\hline \hline
\end{tabular}


In the case of $\mathrm{Li}$, a simple interpolation between the room temperature resistivity at the resistivity at a specified "transition temperature" $T_{\text {tr }}$ is used:

$$
\eta=\left\{\begin{array}{cc}
\eta_{0}+\frac{\eta_{\mathrm{tr}}-\eta_{0}}{T_{t r}} T: & T \leqslant T_{\mathrm{tr}}, \\
\frac{6 \times 10^{-4}}{T^{3 / 2}}: & T>T_{\mathrm{tr}},
\end{array}\right.
$$

where $\eta_{0}=9.4 \times 10^{-8} \Omega \mathrm{m}, \eta_{\mathrm{tr}}=6 \times 10^{-4} / T_{\mathrm{tr}}^{3 / 2} \Omega \mathrm{m}$, and all temperatures are in $\mathrm{eV}$. Where the materials are mixed, the resistivity is found by linearly interpolating between the two resistivities. This was done for the sake of simplicity, but it is clearly somewhat crude. The specific heat capacity used is the same Thomas-Fermi model that Davies uses. ${ }^{30}$

The fast electrons are injected in the first spatial cell on the left-hand end of the grid, and the "laser" intensity is modeled by

$$
I=\beta I_{0} \exp \left[-\left(y-y_{m}\right)^{2} / R^{2}\right]
$$

which is constant over the injection time $t_{\text {pulse }}$. The fast electron temperature $T_{f}$ is determined by Beg's law for $I_{0}$ $<10^{19} \mathrm{~W} \mathrm{~cm}^{-2}$, and by the scaling law of Wilks ${ }^{33}$ for $I_{0}$ $>10^{19} \mathrm{~W} \mathrm{~cm}^{-2}$. The injected fast electron distribution function is specified to be

$$
f_{\text {injected }} \propto \cos ^{M} \theta p^{2} \exp \left(-\sqrt{p^{2}+m_{e}^{2} c^{2}} / k_{\mathrm{B}} T_{f}\right) .
$$

This distribution is a relativistic Maxwellian that is beamed into the target with a divergence half-angle of $\approx 67^{\circ} / \sqrt{M}$.

In the standard run, a uniform spatial grid with 280 cells in $x, 140$ cells in $y$, and a cell size of $0.75 \mu \mathrm{m}$ was used. The momentum grid used 75 grid points and 22 harmonics were used in the expansion. The laser pulse was modeled by setting $I_{0}=5 \times 10^{19} \mathrm{~W} \mathrm{~cm}^{-2}$ and $t_{\text {pulse }}=500 \mathrm{fs}$. The divergence angle was set by $M=4\left(\approx 34^{\circ}\right.$ half-angle). The laser absorption efficiency $\beta$ was set to 0.3 . The simulations were run up to $1 \mathrm{ps}$. The $-\boldsymbol{\nabla} p_{c} / n_{c}$ term is not included in Ohm's law in the standard run.
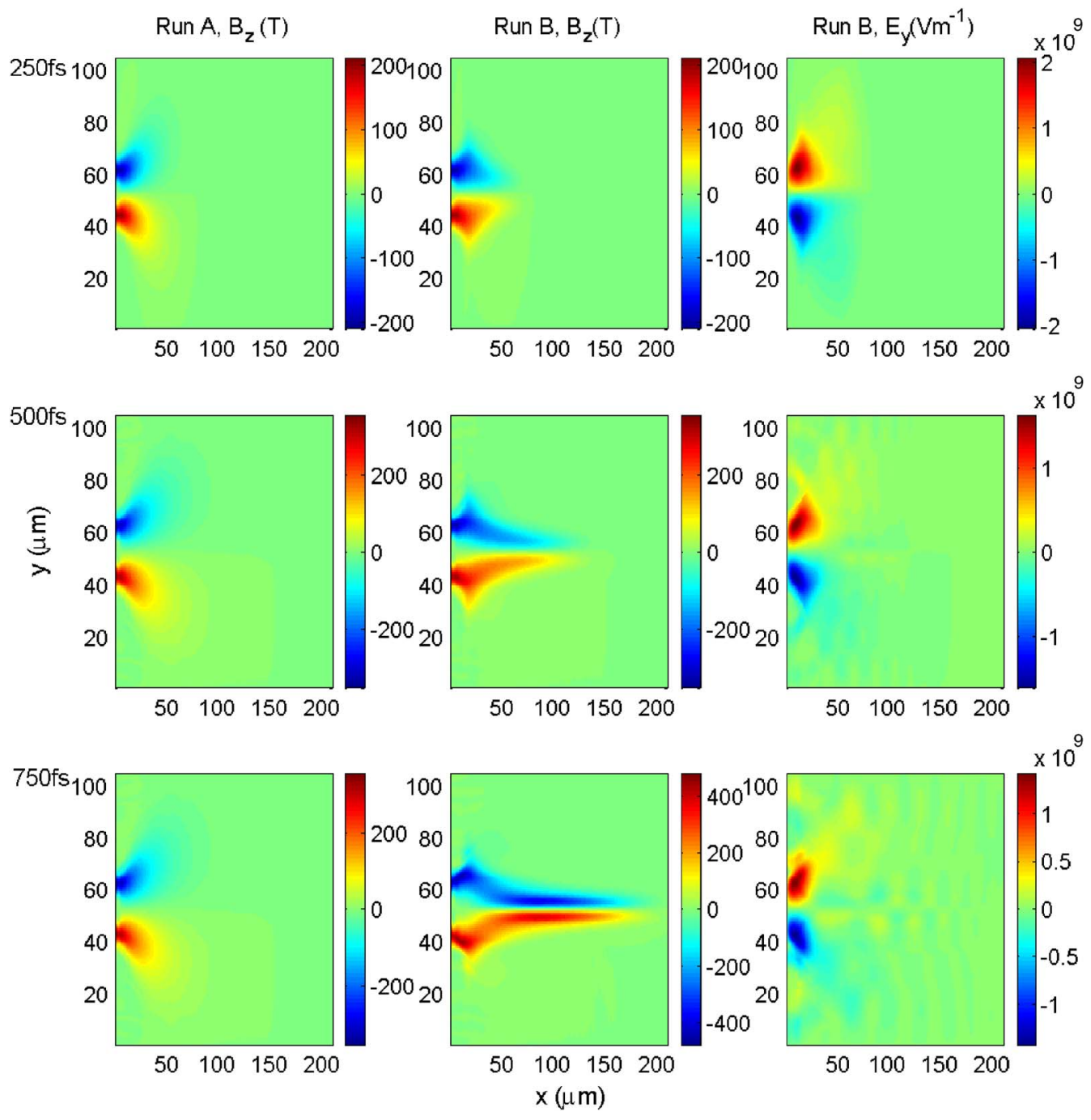

FIG. 3. (Color online) Magnetic field $B_{z}$ (T) in runs A and B at 250, 500, and $750 \mathrm{fs}$ (top-bottom). $E_{y}$ component of electric field in run B also shown. Run $\mathrm{A}$ is the control run. 


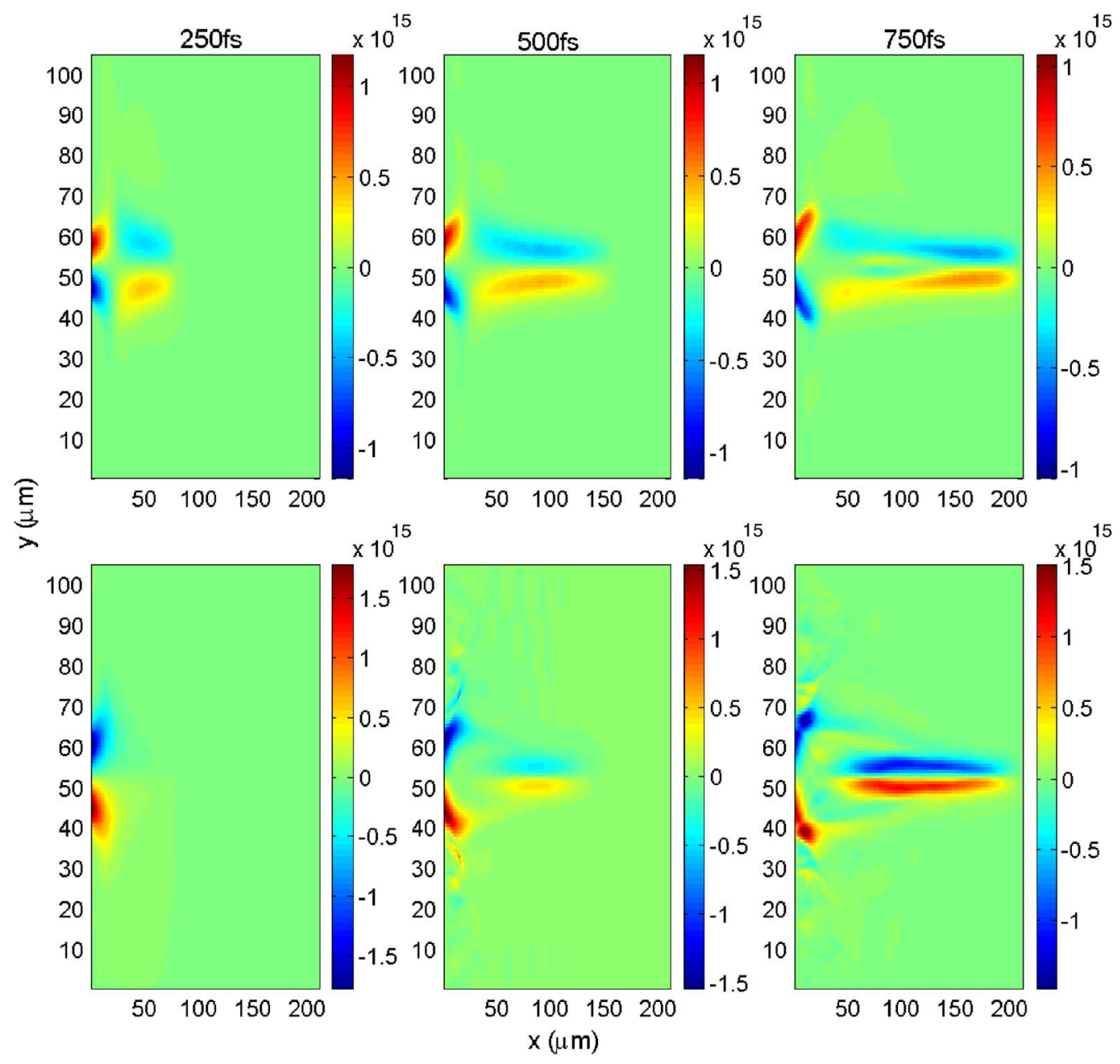

FIG. 4. (Color online) Rate of magnetic field generation $\left(\mathrm{T} \mathrm{s}^{-1}\right)$ in run B due to $\boldsymbol{\nabla} \eta \times \mathbf{j}_{f}$ term (top), and $\eta \boldsymbol{\nabla} \times \mathbf{j}_{f}$ term (bottom) at 250 , 500 , and 750 fs.

\section{RESULTS}

\section{A. Spitzer regime}

A number of runs were carried out based on the standard run to investigate this concept in the regime of Spitzer resistivity. In all of these runs, the initial temperature was set to $200 \mathrm{eV}$. The first run, run A, used a uniform Al target. Run A is therefore the "control" run against which the others are compared. Four runs were carried out using the background structure of Eqs. (7) and (8). These are labeled "B"-"E." Three runs were carried out using the background structure of Eqs. (9) and (8). These are labeled "F"-"H." The geometric parameters used in these runs is given in Table I.

The fast electron density at 750 fs is shown in Fig. 2 for runs A-D alongside a plot of the target $Z$. By comparing the fast electron density in run A to that of runs B-D it is clear that the fast electrons are increasingly collimated as the geometric parameter $B$ is decreased, and that the fast electrons are not collimated in run A. Since all the runs have identical conditions except the target composition, one can only conclude that the target structuring causes the fast electrons to be collimated along the Al fiber. This collimation is done by the generation of a magnetic field. This is shown in Fig. 3 for the case of runs $\mathrm{A}$ and $\mathrm{B}$ at various times. The $E_{y}$ component of the electric field is also shown for run $\mathrm{B}$. This figure shows quite clearly that the collimation is due to the magnetic field, and that the role of the electric field is negligible.

The generation of the collimating magnetic field is due to the interplay of two effects. The first is the expected generation of collimating magnetic field due to the $(\boldsymbol{\nabla} \eta) \times \mathbf{j}_{f}$ term [of Eq. (1)]. This is the term that generates magnetic field that pushes fast electrons towards regions of higher resistivity. This effect should be reinforced by the second effect-the generation of collimating magnetic field due to the $\eta \boldsymbol{\nabla} \times \mathbf{j}_{f}$ term. This is the term that generates magnetic field that pushes fast electrons towards regions of higher fast electron current density. One expects that the first effect will be important at early times and it will be superseded at late times by the second effect (i.e., once collimation has been initiated). When the two terms are plotted at different times, as is done for run B in Fig. 4, this is indeed found to be the case. This is in agreement with the conclusions drawn from analytic model. It was expected that the effect of the $(\nabla \eta)$ $\times \mathbf{j}_{f}$ term would initiate collimation, but it was also expected 

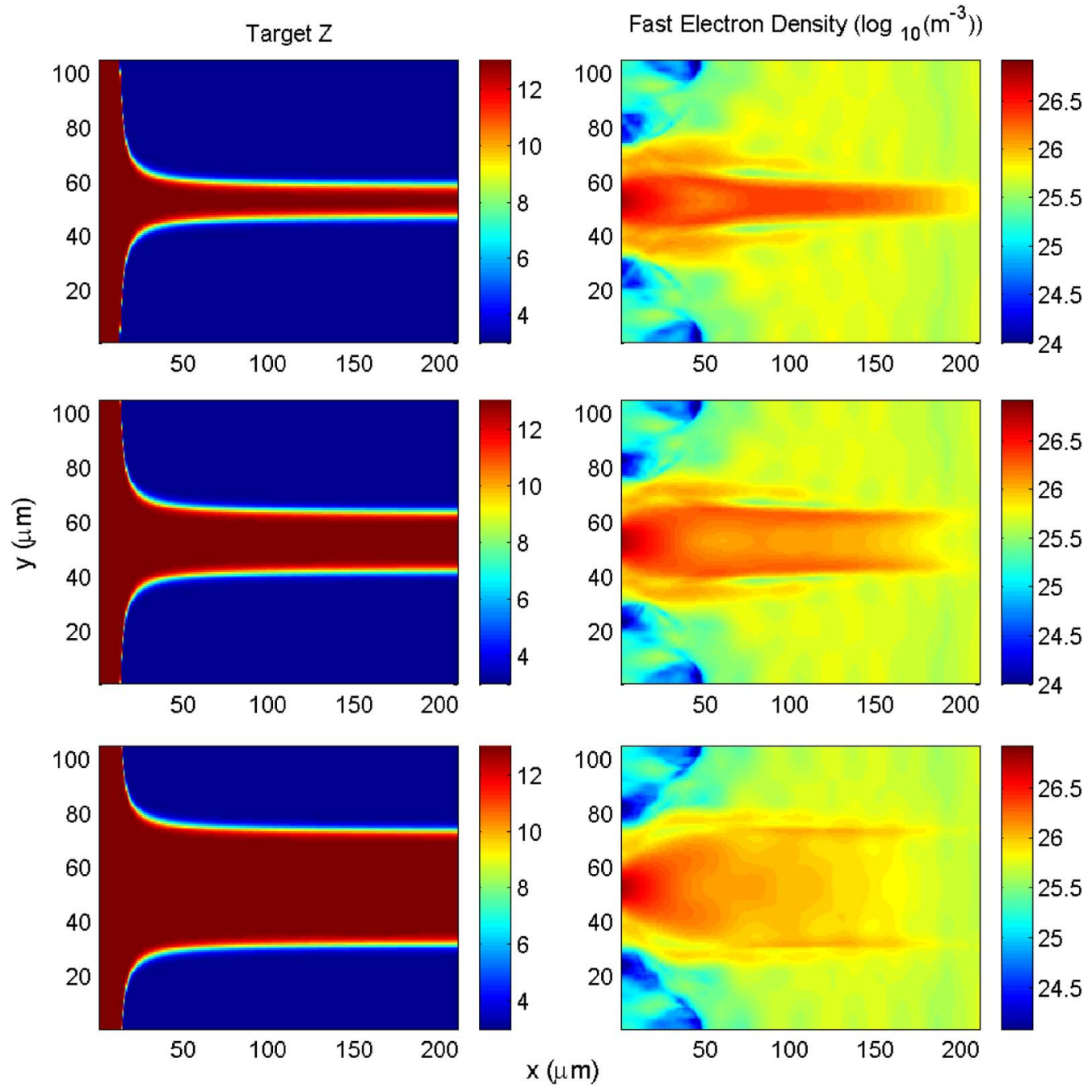

FIG. 5. (Color online) Target $Z$ (left column) and fast electron density at $750 \mathrm{fs}$ (right column) in runs F-H (top-bottom). Runs F-H have their background composition determined by Eq. (9), and the fiber width increases from runs $\mathrm{F}$ to $\mathrm{H}$.

that the effect of the $\eta \nabla \times \mathbf{j}_{f}$ term would be required to enhance the magnetic field enough to provide strong collimation.

Since the generation of collimating magnetic field by the $(\nabla \eta) \times \mathbf{j}_{f}$ term increases with the gradient in target resistivity, the pattern of fast electron flow is different in runs $\mathrm{F}-\mathrm{H}$. In these runs the $\mathrm{Al}$ fibers have sharper boundaries. This results in a stronger, but more a localized magnetic field being generated. This results in slightly better confinement (for a given fiber width) of the fast electrons to the $\mathrm{Al}$ fiber. The fast electron density at 750 fs and the target composition is shown in Fig. 5. It is found that in the wider fibers that the fast electron density peaks at the edges of the fiber. This is the result of the fast electrons residing longer in the magnetic field at the fiber edge while they are deflected.

Runs B and F demonstrate that this scheme can collimate the fast electron beam down to small diameters; for example, in run B the full width at half-maximum (FWHM) of the fast electron beam at $x=100 \mu \mathrm{m}$ and $750 \mathrm{fs}$ is $8 \mu \mathrm{m}$ (4 $\mu \mathrm{m}$ "radius"), and in run F the FWHM at $x=100 \mu \mathrm{m}$ and $750 \mathrm{fs}$ is $12 \mu \mathrm{m}$ (6 $\mu \mathrm{m}$ radius). It should be noted, however, that the transverse beam profile in run B is approximately Gaussian, whereas the beam profile in run $\mathrm{F}$ is much closer to a top-hat profile.

Another run was carried out (run I) that was identical to run $\mathrm{F}$, except that the electron pressure $\left(-\nabla p_{c} / n_{c}\right)$ term was included in Ohm's law. It was found that there were no significant differences between the results of runs I and F. This is expected given that one would estimate that the contribution to the electric field from this term is $\approx 10^{6}-10^{7} \mathrm{~V} \mathrm{~m}^{-1}$.

It is interesting to note that, in the case of a target where the fiber is less resistive than the surrounding material, the same arguments lead to the conclusion that a decollimating magnetic field should be generated which should expel the fast electrons from the fiber. A run was carried out to examine this (run J). Plots of target $Z$, the $B_{z}$ component of the magnetic field, and the fast electron density at $750 \mathrm{fs}$ are shown in Fig. 6. The target $Z$ was chosen to be the "inverse" of that in run F. The run is otherwise identical to the standard run. Figure 6 shows that although some fast electrons are able to enter the fiber, most of the fast electrons are expelled from the fiber. This also shows that it is done by a strong 


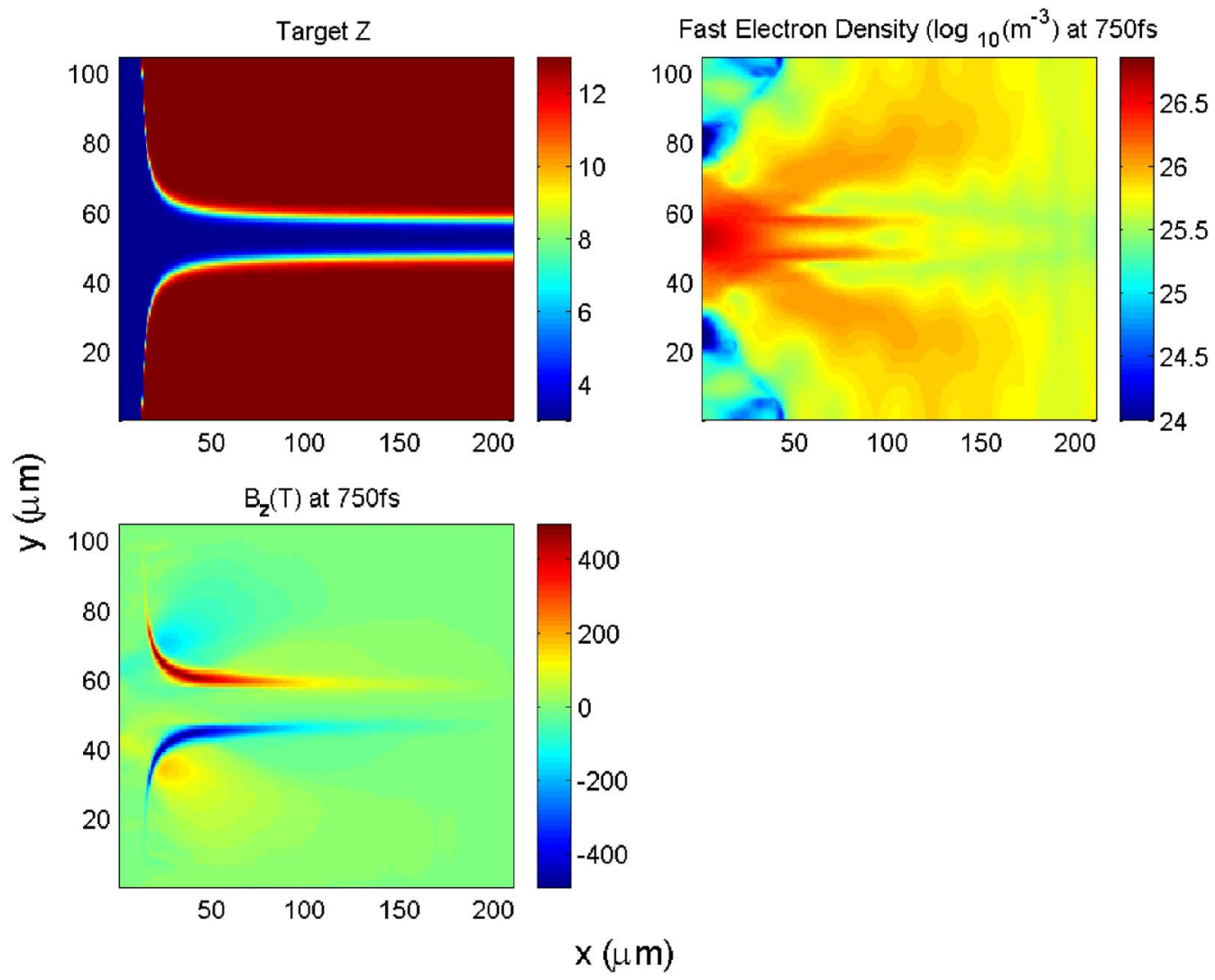

FIG. 6. (Color online) Target $Z$ (top left), fast electron density at $750 \mathrm{fs}$ (top right), and $B_{z}$ (T) at $750 \mathrm{fs}$ (bottom left) in run J. Run J is identical to run F except that the material composition has been "reversed" so the fiber is now less resistive than the cladding material.

decollimating magnetic field. This demonstrates that the theory also makes the correct prediction for the "inverse" case as well.

\section{B. Cold target regime}

One expects the target to collimate the fast electrons even when the target is initially cold provided that the resistivity of the $\mathrm{Li}$ is always lower than that of Al. Using the intialization for run $\mathrm{F}$ and the cold target resistivity described in Sec. III, three runs were carried out (labeled "K," "L," and "M"). For the resistivity model of Li we used transition temperatures $\left(T_{\text {tr }}\right)$ of $100 \mathrm{eV}$ (run K), $50 \mathrm{eV}$ (run L), and $25 \mathrm{eV}$ (run M). The resistivity against temperature is plotted in Fig. 7. From Fig. 7, it can be seen that one expects runs $\mathrm{K}$ and $\mathrm{L}$ to exhibit collimation, but what happens in run $\mathrm{M}$ is much more uncertain.

In the simulations, it is found that collimation occurs in all three runs. This is shown in Fig. 8. However, in the case of run $\mathrm{M}$, there is clearly a transient phase in which the magnetic field acts to expel fast electrons from the fiber instead of collimating them. This is expected since from the resistivity curves shown in Fig. 7 , it is clear that there is a temperature range over which the resistivity of $\mathrm{Li}$ is greater than that of $\mathrm{Al}$ in run $\mathrm{M}$. The target rapidly heats up enough to pass through this transient regime, and therefore the collimation of the beam still occurs, albeit some fast electrons at the head of the beam are lost. This produces the "wing" features in the fast electron density plot of run M (see Fig. 8).
This set of runs show that this collimation scheme should still work even when the target is initially at a low temperature. Furthermore, it shows that the collimation scheme can still work even if there is a small temperature range over which the material surrounding the fiber has a higher resistivity than the fiber. This collimation scheme

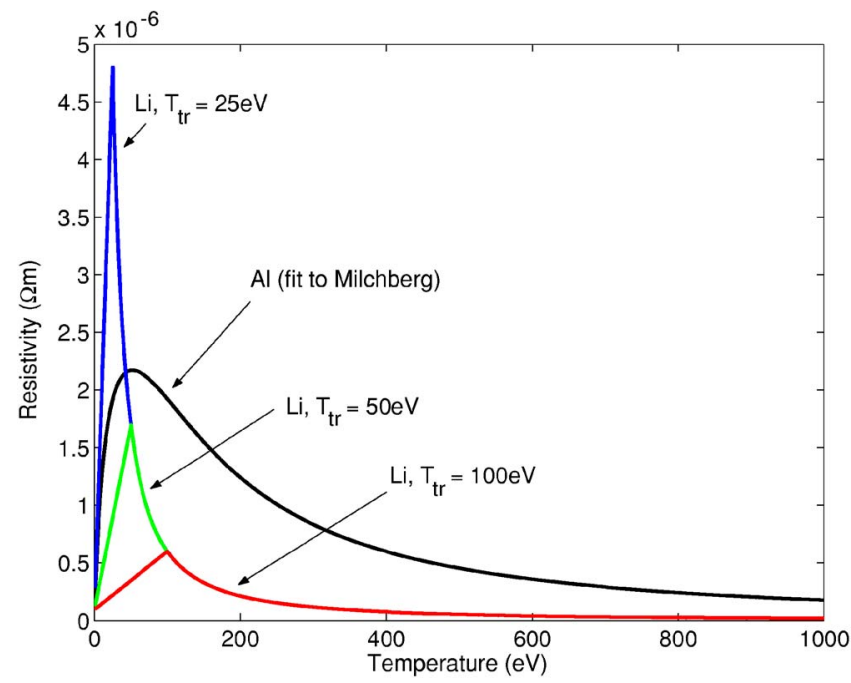

FIG. 7. (Color online) Resistivity vs temperature profiles used in cold target simulations: Fit to Milchberg for $\mathrm{Al}$ (black), $\mathrm{Li}$ for $T_{\mathrm{tr}}=25 \mathrm{eV}$ (blue), $\mathrm{Li}$ for $T_{\mathrm{tr}}=50 \mathrm{eV}$ (green), Li for $T_{\mathrm{tr}}=100 \mathrm{eV}$ (red). 


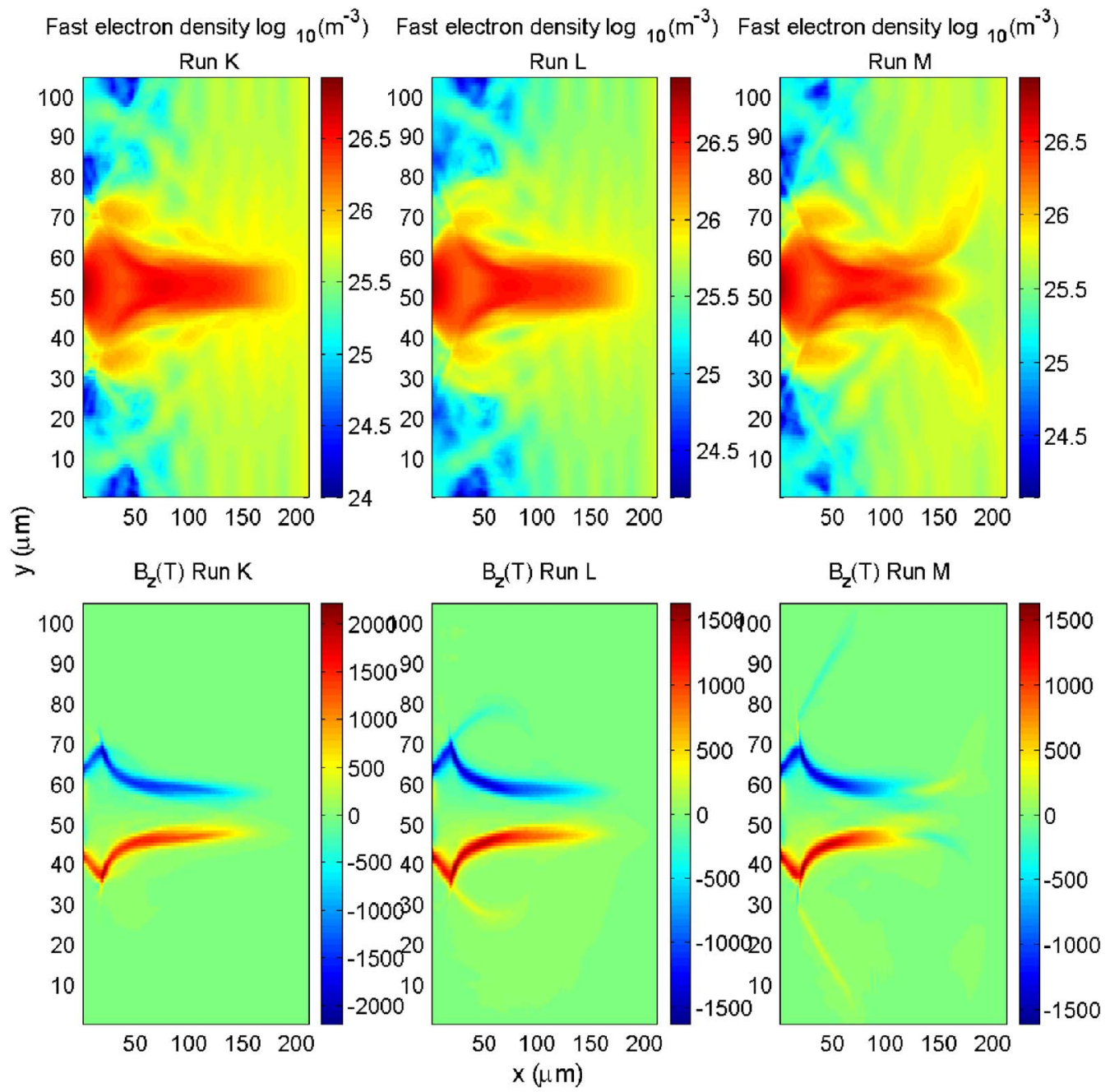

FIG. 8. (Color online) Fast electron density $\left[\log _{10}\left(m^{-3}\right)\right]$ and magnetic field $\left[B_{z}(\mathrm{~T})\right]$ at $750 \mathrm{fs}$ in runs K, L, and M. These are the "cold start" runs.

therefore seems to be robust with respect to material properties at low temperatures.

\section{SUMMARY AND CONCLUSIONS}

A scheme for collimating a beam of fast electrons, which are produced by irradiation with an ultraintense laser pulse, in a specially engineered solid target has been proposed. The target is engineered such that it consists of a fiber surrounded by material of a lower resistivity than the fiber. The fast electrons are collimated along the fiber by a magnetic field that is generated by the fast electron current.

An analytic theory was presented that demonstrated that this would produce significant magnetic field generation. $\mathrm{Nu}-$ merical calculations carried out using the hybrid VFP code LEDA showed that this concept can work, both in the regime of Spitzer resistivity (where the target is initially hot), and in the regime where the target is initially cold. Importantly, we showed that the scheme still works well in the case where there is a small temperature range in which the resistivity of the material surrounding the fiber is greater than that of the fiber itself. The numerical calculations also showed that the fast electron beam could be collimated down to radii of $6 \mu \mathrm{m}$, and that the best results are achieved when there is a sharp transition between the two materials. This approach is therefore at least as good as that proposed by Campbell ${ }^{27}$ or Kodama ${ }^{28}$ if not better in this regard. Since this method does not rely on vacuum gaps it may be better suited to some applications. This may find application in $K_{\alpha}$ radiography, and in FI. It may be possible to incorporate this into a FI scenario in a similar way to that proposed by Campbell et al.; i.e., by including a collimator segment behind the cone tip. Care needs to be taken when choosing materials, as the need to produce a large resistivity gradient in the radial direction must be balanced against choosing a fiber with such a high resistivity that a lot of fast electron energy is lost due to the resistively generated electric field along the fiber. It should be noted, however, that this was not a significant issue in the case considered in this paper.

\section{ACKNOWLEDGMENTS}

The authors are grateful for the use of computing resources provided by the Science and Technology Facilities Council's e-Science facility.

\footnotetext{
${ }^{1}$ M. Tabak, J. Hammer, M. E. Glinsky, W. L. Kruer, S. C. Wilks, J. Woodworth, E. M. Campbell, M. D. Perry, and R. J. Mason, Phys. Plasmas 1, 1626 (1994).
} 
${ }^{2}$ M. Tabak, D. S. Clark, S. P. Hatchett et al., Phys. Plasmas 12, 057305 (2005).

${ }^{3}$ H.-S. Park, D. M. Chambers, H.-K. Chung et al., Phys. Plasmas 13, 056309 (2006).

${ }^{4}$ R. A. Snavely, M. H. Key, S. P. Hatchett et al., Phys. Rev. Lett. 85, 2945 (2000).

${ }^{5}$ E. L. Clark, K. Krushelnick, J. R. Davies et al., Phys. Rev. Lett. 84, 670 (2000).

${ }^{6}$ M. S. Wei, J. R. Davies, E. L. Clarke et al., Phys. Plasmas 13, 123101 (2006).

${ }^{7}$ F. N. Beg, A. R. Bell, A. E. Dangor et al., Phys. Plasmas 4, 447 (1997).

${ }^{8}$ K. Tanaka, R. Kodama, H. Fujita et al., Phys. Plasmas 7, 2014 (2000).

${ }^{9}$ J. Fuchs, T. E. Cowan, P. Audebert et al., Phys. Rev. Lett. 91, 255002 (2003).

${ }^{10}$ L. Gremillet, F. Amiranoff, S. D. Baton et al., Phys. Rev. Lett. 83, 5015 (1999).

${ }^{11}$ F. Pisani, A. Bernardinello, D. Batani et al., Phys. Rev. E 62, R5927 (2000).

${ }^{12}$ D. Batani, A. Antonicci, F. Pisani et al., Phys. Rev. E 65, 066409 (2002).

${ }^{13}$ W. Theobald, K. Akli, R. Clarke et al., Phys. Plasmas 13, 043102 (2006).

${ }^{14}$ M. H. Key, M. D. Cable, T. E. Cowan et al., Phys. Plasmas 5, 1966 (1998).

${ }^{15}$ E. Martinolli, M. Koenig, S. D. Baton et al., Phys. Rev. E 73, 046402 (2006).

${ }^{16}$ J. R. Davies, A. R. Bell, and M. Haines, Phys. Rev. E 56, 7193 (1997).

${ }^{17}$ J. R. Davies, Phys. Rev. E 68, 056404 (2003).
${ }^{18}$ R. J. Mason, E. S. Dodd, and B. J. Albright, Phys. Rev. E 72, 015401(R) (2005).

${ }^{19}$ R. J. Mason, Phys. Rev. Lett. 96, 035001 (2006).

${ }^{20}$ R. G. Evans, E. L. Clark, R. T. Eagleton et al., Appl. Phys. Lett. 86, 191505 (2005).

${ }^{21}$ J. J. Honrubia, A. Antonicci, and D. Moreno, Laser Part. Beams 22, 129 (2004).

${ }^{22}$ R. P. J. Town, C. Chen, L. A. Cottrill et al., Nucl. Instrum. Methods Phys. Res. A 544, 61 (2005).

${ }^{23}$ T. Taguchi, Jr., C. S. Liu, K. Mima et al., Phys. Rev. Lett. 86, 5055 (2001).

${ }^{24}$ R. Stephens, R. A. Snavely, Y. Aglitskiy et al., Phys. Rev. E 69, 066414 (2004).

${ }^{25}$ K. Lancaster, J. S. Green, D. S. Hey et al., Phys. Rev. Lett. 98, 125002 (2007).

${ }^{26}$ A. R. Bell and R. J. Kingham, Phys. Rev. Lett. 91, 035003 (2003).

${ }^{27}$ R. B. Campbell, J. S. DeGroot, T. A. Mehlhorn, D. R. Welch, and B. V. Oliver, Phys. Plasmas 10, 4169 (2003).

${ }^{28}$ R. Kodama, Y. Sentoku, Z. L. Chen et al., Nature 432, 1005 (2004).

${ }^{29}$ J. R. Davies, J. S. Green, and P. A. Norreys, Plasma Phys. Controlled Fusion 48, 1181 (2006).

${ }^{30}$ J. R. Davies, Phys. Rev. E 65, 026407 (2002).

${ }^{31}$ A. R. Bell, A. P. L. Robinson, M. Sherlock, R. J. Kingham, and W. Rozmus, Plasma Phys. Controlled Fusion 48, R37 (2006)

${ }^{32}$ A. P. L. Robinson, A. R. Bell, and R. J. Kingham, Plasma Phys. Controlled Fusion 48, 1063 (2006)

${ }^{33}$ S. C. Wilks and W. L. Kruer, IEEE J. Quantum Electron. 33, 1954 (2000). 